\title{
OPTIMIZATION STRATEGIES TO ELIMINATE INTERFACE CONFLICTS IN COMPLEX SUPPLY CHAINS OF CONSTRUCTION PROJECTS
}

\author{
Qianqian JUa, b, Lieyun DINGa, Miroslaw J. SKIBNIEWSKI ${ }^{\text {b, c, d }}$ \\ ${ }^{a}$ School of Civil Engineering \& Mechanics, Huazhong University of Science and Technology, \\ Wuhan, Hubei 430074, China \\ ${ }^{b}$ Department of Civil \& Environmental Engineering, University of Maryland, \\ College Park, MD 20742-3021, USA \\ cInstitute for Theoretical and Applied Informatics, Polish Academy of Sciences, Gliwice, Poland \\ ${ }^{d}$ Chaoyang University of Technology, Taichung, Taiwan
}

Received 10 Nov 2015; accepted 18 Aug 2016

\begin{abstract}
The supply chains of complex construction projects are characterized by multi-disciplinary participants, adversarial short-term relationships and fragmentations in project delivery procedures. Multi-disciplinary participants tend to make decisions on project activities based on their own objectives and value systems independently with little regard of holistic project performance. The lack of common values results in a limited understanding of how behaviors of one discipline impact on the related disciplines. This has led to tons of interface conflicts which ultimately affect project effectiveness. This paper investigates this problem by discussing Interface Value for the core players to understand the value-driven behaviors on the boundaries among owner and multiple prime contractors in complex construction projects. Two types of interface conflicts are recognized and defined. Value Optimization Strategy is proposed under status quo delivery methods by adjusting and reallocating interface responsibilities between related contractors. Improvements of procurement procedures are the key solutions to interface conflicts. Prospects of integrated project delivery (IPD) in eliminating interface conflicts are also discussed. The paper concludes that Value Optimization Strategy and IPD-based approach are expected to eliminate interface conflicts of complex construction projects, integrate the complex supply chain and lower the potential risks for project delivery delay and cost overrun.
\end{abstract}

Keywords: construction industry, interface management, project management, supply chain, integration, conflict, IPD.

\section{Introduction}

Complex projects are a set of projects that share particular defining characteristics: they are high-tech, capital intensive engineering projects that are of a significant scale, relatively long duration, and require firms to work collaboratively across firm boundaries in project delivery (Whyte et al. 2016). Large-scale engineering, construction and infrastructure projects are complex and notoriously difficult to manage. In the construction sector, the supply chain network can often be extremely complex, particularly on a larger project where the number of separate supplying organizations could number many hundreds (Dainty et al. 2001). Complex construction projects deliver various subsystems. Their deliveries require subsystems integration capabilities, as the subsystems of complex construction projects are designed with functional linkages and integrated through a supply chain network of component, service and subsystem suppliers. As with the improvement of automation in construction industry, the number of functional linkages among sub- systems has increased dramatically and the projects tend to be increasingly complex. Thus, owners/project managers must deal with more complex interfaces than ever before (Siao, Lin 2012).

Meanwhile however, the construction industry is arguably the least integrated of the all the major industrial sectors, characterized by adversarial practices, disjointed supply relationships and a lack of trust between clients, main contractors and subcontractors (Fearne, Fowler 2006). The products of construction industry are partly carried out on a temporary site by a temporary organization made up of different parties (developers, designers, contractors and suppliers), which comes to an end after completion, bringing added uncertainty and complexity to this industry (Arantes et al. 2015). The multi-disciplinary participants get involved into project activities at different construction phases to deliver the holistic system as final production functioning with various subsystems working together. Interface issues come from different 
understandings of associated project activities and developments of value systems over their own domains of interface responsibility. Perception of value is individual and personal, and is therefore subjective (Emmitt et al. 2005). Hence, objectives and values differ from discipline to discipline. The multi-disciplinary participants develop their own goals and value systems. Each discipline has become dedicated to the optimization of its own function with little regard, or understanding of, its effect on the performance of the construction process (Gunasekaran, Love 1998). The interfaces between functional disciplines have become a potential barrier to effective communication and coordination of construction projects (Love, Gunasekaran 1997).

Further problems are associated with complex supply networks including multiple customer-supplier relationships as well as supplier-supplier relationships. In addition to interfaces among disciplines inside civil construction sectors, there are huge amount of interfaces between civil construction sectors and equipment sectors of complex construction projects. The integral supply chains of complex construction projects have the characteristics of both manufacturing and construction industry. As a consequence, it is usual for the industry to suffer considerable losses caused by conflicts, budget overruns, claims and counter claims, which are mainly caused by supply delays and disruptions (Arantes et al. 2015). The failure of complex supply chains to meet client demands is characterized by adversarial short-term relationships, multidisciplinary participants and fragmentations in project delivering procedures. Although overall effectiveness of complex supply chains depends on collective performance of all the subsystems, the behaviors of the independently managed project participants who seek to maximize their own profits may conflict and result in inefficiencies of the entire supply chain. In real projects, the way contractors traditionally perform (without common goals and coordinated objectives) the project delivery outcomes remains the same as before which means they are only responsible for the owner with no contractual relationship between each other. And it remains quite common that the owners of complex projects in construction industry who are supposed to be responsible for the interface issues are inexperienced in supply chain coordination and lack effective interface management (IM) approaches.

The lack of common objectives and values among multi-disciplinary participants of complex construction projects result in a limited understanding of how behaviors of one discipline impact on other project participants involved in the same activities and ultimately leads to unnecessary interface conflicts and affects project effectiveness. Construction management should see benefits in construction projects when interfaces are well managed and the IM is applied and well implemented (Siao, Lin 2012). The aim of this paper is to eliminate unnecessary waste in interface conflicts. Interface Value for the core players is discussed to help understand the value-driven behaviors on the boundaries among the owner and multi- ple prime contractors and how behaviors of one discipline impact the value of the entire supply chain. Value Optimization Strategy and an IPD-based strategy are proposed. Prospects of these strategies in eliminating interface conflicts are analyzed, validated and concluded.

\section{Literature review}

The existing literature offers various dimensions in understanding the fragmentation nature of construction industry and seeks to eliminate conflicts in the complex supply chains. "Supply Chain Integration" and "Interface Management" are two useful dimensions among existing researches, but few studies have focused on directly understanding and avoiding value-driven conflicting behaviors on the interfaces of multiple disciplines by combining both of these two dimensions.

Supply chain management (SCM) has originated and flourished in the manufacturing industry. Project SCM in construction can be defined as "the network of facilities and activities that provide customer and economic value to the functions of design development, contract management, service and material procurement, materials manufacturing and delivery, and facilities management" (Love et al. 2004). A growing body of research in construction supply chain (CSC) is developing, with a view to transferring and updating the SCM concepts successfully applied in the manufacturing industries to the construction sector (Segerstedt et al. 2010a, 2010b; Fearne, Fowler 2006). Among these researches, calls for improved collaboration, integration, communication and coordination between customers and suppliers throughout the project supply chain have inspired numerous researches to eliminate conflicts and remedy the fragmentation nature of construction industry. Love et al. (2004) presented a seamless project SCM model to integrate the design and production process on the customer-supplier interface. Segerstedt et al. (2010a) emphasize the importance of "supply chain integration" and direct the attention towards the different types of interdependencies existing in construction supply chains. Humphreys et al. (2003) develop a procurement approach in order to improve the relationships between main contractor and subcontractors. Briscoe and Dainty (2005) identify the key factors for successful integration of the construction supply chain through case study. Segerstedt et al. (2010b) seek to improve construction supply chain collaboration and performance through a lean construction pilot project. However, recent research shows that the global awareness level of SCM and the relationship level among entities in the construction industry are still low (Arantes et al. 2015). Fearne and Fowler (2006) argue that a change in the management of relationships among customers, contractors, sub-contractors and suppliers is essential in order to improve the effectiveness and efficiency of CSC. Interface management (IM) is the management of interface problems involving people, components, systems and concepts (Nooteboom 2004). Unaware of SCM concepts, some construction literatures discussed "supply chain 
integration" related issues through IM perspective in different fields of construction industry and explored possible causes and solutions. Interface problems between particular constructions parties were identified and assessed (Al-Hammad 1995, 2000). Besides interface issues among people, some researchers explored how management of organizational integration could improve project to-project and project-to-organization interfaces (Turkulainen et al. 2015). Each construction project that is characterized by extreme complexity and non-standardized production differs in that it is designed and executed to meet owners' needs (Lin 2013). Therefore, interface problems are always specifically defined and discussed based on particular project elements such as delivery methods and types of the construction projects and the solutions for interface conflicts are not common. Chan et al. (2005) proposed a four-step conceptual interface management procedure and proposed an IM framework for Chinese BOT Projects. Pavitt and Gibb (2003) categorized the three different types of interface management and presented a standardized strategy for cladding interfaces to avoid the endemic interface problems occurring on-site. Other researcher have proposed approaches to support IM, such as the novel Multilevel Interface Matrix approach to enhance interface management during the construction phase (Siao, Lin 2012) and the process to identify the critical "Interfaces" in a process safety management system (Kelly, Berger 2006). Also, IT related approaches have been explored and applied to improve IM efficiency. Senthilkumar et al. (2010) proposed a Design Interface Management System to formulate the Dependency Structure Matrix on large construction projects. Senthilkumar and Varghese (2012) evaluated the effectiveness of "Design Interface Management System" methodology and its implementation on the construction design processes. Lin developed a web-based platform for communicating interfaces among all project participants (Lin 2013). Ju and Ding (2014) developed a web-based integrated interface management system for standardized interface information presentation, storage and exchanging, effective interface conflicts coordination and interface information tracking.

Despite the literature mentioned above which addressed some SCM approaches and IM solutions for fragmentations and inefficiencies in construction industry, there is still lack of concern about the interface conflicts among multi-disciplinary participants in complex construction projects which are characterized by adversarial short-term relationships and fragmentations in project procurement procedures from the perspective of SCM.

\section{Background of interface conflicts in complex construction projects}

\subsection{Two types of interface conflicts}

Interface conflicts are common in construction industry due to the following reasons: (1) temporary organi- zations; (2) low-bid strategy of the owner; (3) no contractual relationships and common goals among prime contractors; (4) different understandings of tasks and actions; (5) fuzziness and inconsistencies of contractual responsibilities; (6) the historical fragmentations of project delivery systems (Ju, Ding 2014; Arantes et al. 2015; Al-Hammad 1995, 2000). As a result, numerous interface conflicts occurred in different phases of complex construction projects and have led to unnecessary waste including reworks, delays and failures: (1) Design stage: delay or poor quality of the deliveries of functional linkages' design material and information exchange; insufficient interface information communication in coordinated design environment which leads to reworks and failures. (2) Manufacturing \& Construction stage: gaps between design and construction; functional linkages' design changes of related subsystems which lead to interface responsibility conflicts among prime contractors; components' space conflicts; working face conflicts; equipment technical parameter conflicts; late delivery or poor quality of upstream project tasks. (3) Acceptance stage: the omission in subsystem acceptance which is one of the major causes of errors and reworks in integrated acceptance and project final acceptance; errors of functional linkages during integrated acceptance which lead to reworks and waste of multiple prime contractors (Nooteboom 2004; Harrison 2004; Al-Hammad 2000). Project activities are schedule driven. Given a well-structured schedule, if everyone stays on their part of the schedule, the work flows smoothly and maximum performance is achieved (Ballard, Howell 1995). The other cause of interface conflicts in complex construction projects lies in unreliability of interface tasks. Hence, two types of interface conflicts are recognized and defined based on the research scope of this paper. Interface Conflict Type I is inconsistency of interface related overlapping terms of the contracts between the owner and the contractors on different sides of the "interfaces" terms which compose of two types: the interface responsibilities that should be taken by downstream contractor but are only clearly stated in contract of the upstream contractor and the interface responsibilities that should be taken by upstream contractor but are only clearly stated in contract of downstream contractor. In real projects, only the latter have direct impact on project effectiveness, so only the latter are considered in this paper. Interface Conflict Type II is the newly appearing interface tasks which are always only become apparent at the later stages of projects (Harrison 2004).

\subsection{Interface events and interface tasks}

A Chain is only as strong as its weakest link - "Interface". IM is a relatively new topic in construction industry. It is not uniquely defined and usually varies based on an industry's characteristics and management needs (Ju, Ding 2014). In offshore construction, interface is defined as "the common boundaries between people, systems, equipment, or concepts" (Nooteboom 2004); the defini- 
tion of interface for the overall railroad and rail transit project is "a common boundary or interconnection between systems, equipment concepts or human beings" (Harrison 2004); in civil construction, interface is defined as "the communication, coordination and responsibility across a common boundary between organizations, phases, or physical entities which are interdependent" (Wideman 2002). The difference between the definitions of "Interface" in various industries relies on the artificial divisions of project management elements with the similar goal - reducing conflicts and improving integration from system perspective. The definition of "Interface" is broad and it is necessary to narrow the scope of this paper from the very beginning. The focus of this paper is the interface conflicts on the boundary of multi-disciplinary participants of complex construction projects. The basic way of interface management in complex construction projects is that the project manager schedules and controls the key interface events according to system's general design, while the responsible subsystem contractors manage the tasks or work included in these interface events. The following discussion about interface value starts with abstracting the concept of "Interfaces" to two key elements in different layers: "Interface Events" and "Interface Tasks". An interface event is a set of project activities (interface tasks on the boundaries between multiple prime contractors) which are performed to realize some certain functional linkages among various disciplines (see Fig. 1).

\subsection{Status quo interface events}

An interface event is composed of a certain amount of interface tasks performed by different sub-system contractors in a specific order. Without considering parallel relationships, the upstream entity of an interface event is defined as upstream contractor which is responsible for the preceding tasks of the interface event while the related downstream entity is defined as downstream contractor. Interface conflicts always only become apparent at the later stages of the project. Hence, within the range of an interface event, downstream contractor always find frequent interface conflicts after the delivery of interface tasks from upstream contractors. Since the solutions of interface conflicts can only be accomplished within tight time constraints due to looming project deadlines, downstream contractor will hold interface meetings as soon as

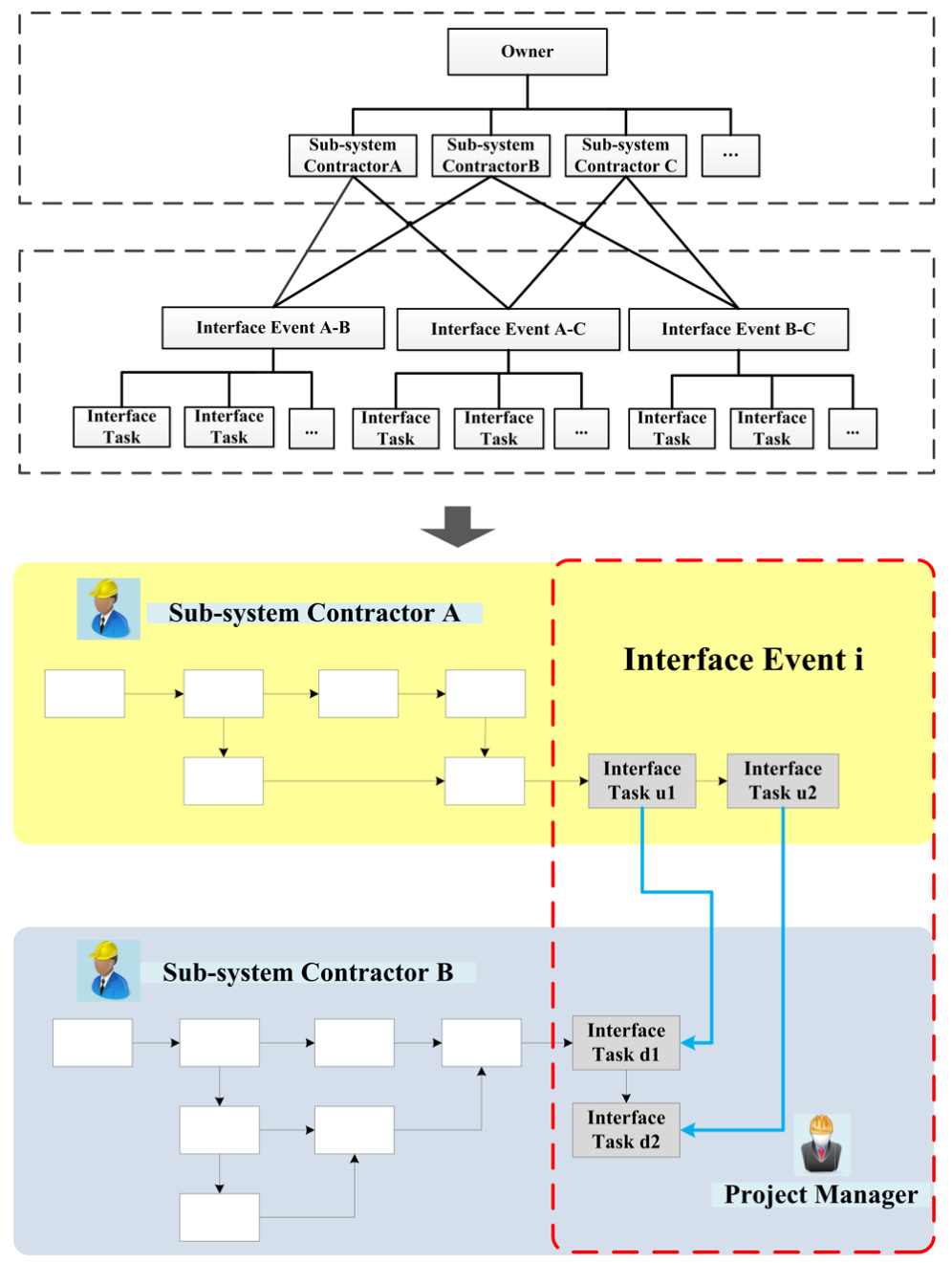

Fig. 1. Interface events and interface tasks 


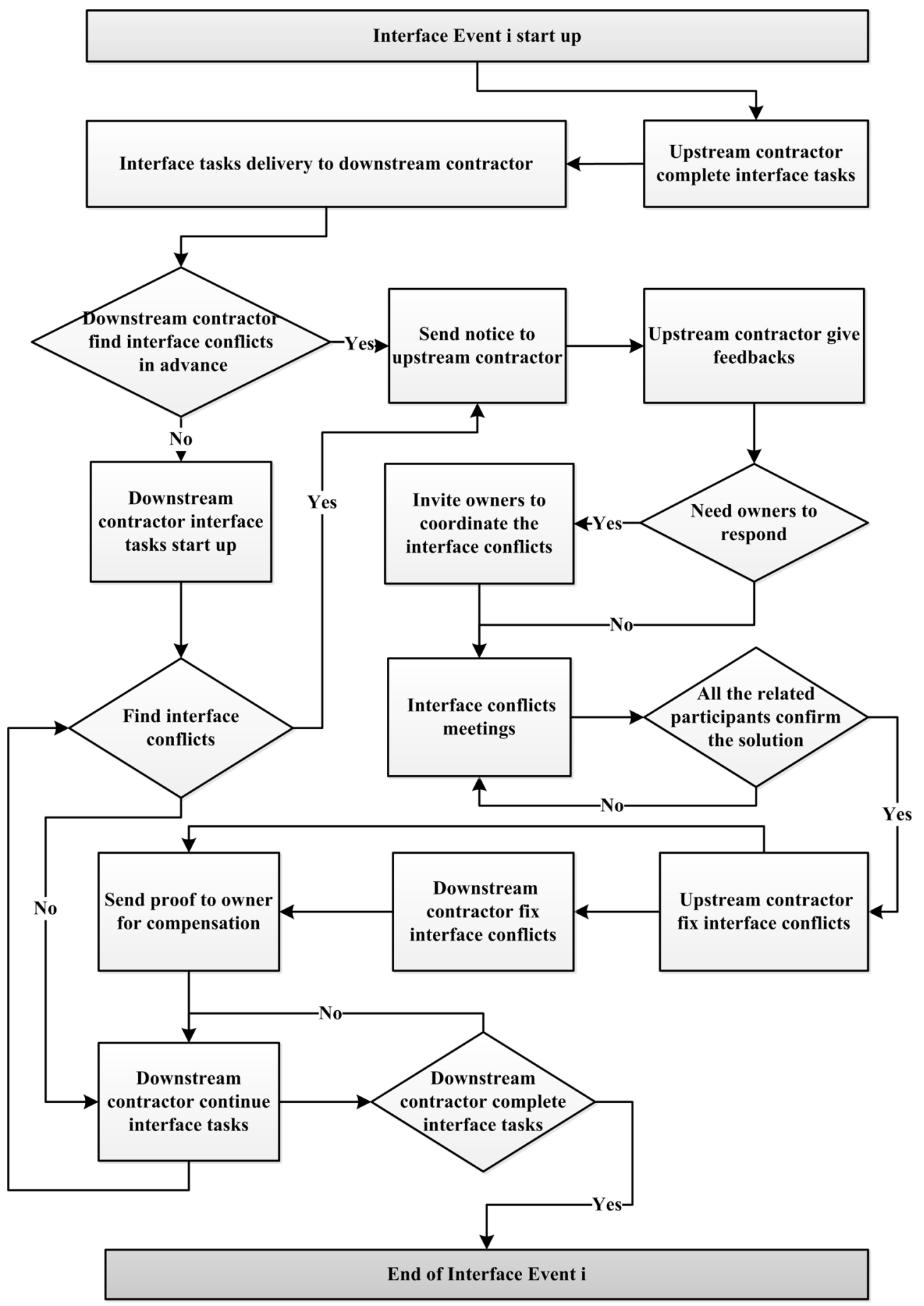

Fig. 2. Status quo interface event workflow

possible with upstream contractor as well as the owner if necessary. However, the delivery procedures of complex construction projects are fragmented and most of the functional linkages are only explicitly manifested in the delivery of disciplines that the downstream contractors are responsible for. The upstream contractors tend to reluctantly response to the interface conflicts resolutions to minimize their cost. Iterative processes of fixing the interface conflicts in interface invents generate unnecessary waste of all the related project participants as well as the entire supply chain (see Fig. 2).

\section{Interface Value for the core players in the complex supply chain}

Value is what an individual or organization places on a process and the outcome of that process. According to AS/NZS 4183 (1994), Value Management (VM) is defined as "a structured and analytical process, the purpose of which is to seek to achieve value for money by providing all of the necessary functions at the lowest cost consistent with required levels of quality and performance" (AS/NZS 4183 1994). Participants' behaviors in interface 
events of complex supply chains are value driven. We define a function " $V$ " (Interface Value) to describe the value of interface events. Discussing the gaps among "Interface Value" from different participants' perspectives is essential in understanding of how behaviors of one discipline impact on other project participants involved in the same interface event and eliminating interface conflicts. Under a short-term cooperation environment, the discussion begins with a normal statement of the basic goal from the contractor's point of view: to maximize the profit of interface tasks in the interface events. However, from the owner's point of view, the basic goal is to minimize the cost of interface events which is the total cost of all the interface tasks with the completion of required functional linkages. Assuming that: there are $\mathrm{N}$ interface events between Contractor A and Contractor B; the upstream entity of an interface event $i$ is Contractor A while the related downstream entity is Contractor B which means that the interface tasks of Contractor A are preceding tasks. For the sake of simplicity, only two contractors and the owner are considered in the model and the upstream-downstream relationships of the interface tasks of the two contractors always remain constant in interface events.

\subsection{Upstream contractor}

For Contractor A, the value of interface events can be represented by the profit earned in upstream interface tasks. So Interface Value, $V_{U}$, for upstream contractor is given by:

$$
V_{U}=\sum_{i}\left(P_{U i}-U_{i}-W_{U i}\right),
$$

where $V_{U}$ is interface tasks' value for the upstream contractor; $P_{U i}$ is the price of upstream interface tasks in interface event $i ; \quad U_{i}$ is the cost for upstream interface tasks actually performed before delivering interface tasks to downstream contractor in interface event $i ; W_{U i}$ is the cost for interface conflicts for upstream contractor in interface event $i$. Specifically, according to status quo interface event workflow (see Fig. 2) $U_{i}$ and $W_{U i}$ are given by:

$$
\begin{gathered}
U_{i}=M_{U i} C_{U i} \\
W_{U i}=R_{U i}\left(k_{i}-H_{i}\right)=\sigma_{i}\left(S_{i}-M_{U i}-M_{D i}\right)\left(k_{i}-H_{i}\right),
\end{gathered}
$$

where: $M_{U i}$ - upstream interface tasks performed before delivering interface tasks to downstream contractor; $\mathrm{M}_{D i}$ - downstream interface tasks performed in interface event $i$ as planned; $\mathrm{S}_{i}$ - actually required interface tasks in interface event $i ; C_{U i}$ - unit cost for upstream interface tasks in interface event $i ; R_{U i}$ - upstream reworks for interface conflicts in interface event $i ; k_{i}$ - unit cost for reworks for interface conflicts claimed from downstream for interface event $i ; \sigma_{i}$ - proportion of responsibilities for interface conflicts claimed from owner and upstream contractor in interface meetings for interface event $i ; H_{i}$ - offset unit cost in compensation of interface event $i$. In addition to the cost for upstream interface tasks actually performed before delivering interface tasks to downstream contractor, the cost for interface conflicts would arise to upstream contractor if downstream contractor find them and initiate interface meetings to discuss the allocation of responsibilities for the interface conflicts in the latter processes of interface event $i$. The amount of upstream reworks $R_{U i}$ would be negotiated in interface meetings depending on the proportion of responsibilities for interface conflicts claimed from owner and upstream contractor and the amount of interface conflicts. The actual unit cost for upstream reworks would be partly offset through compensation from the owner at the end of the project.

\subsection{Downstream contractor}

For Contractor B, the value of interface events is represented by the profit earned in downstream interface tasks. So Interface Value, $V_{D}$, for downstream contractor is given by:

$$
V_{D}=\sum_{i}\left(P_{D i}-D_{i}-W_{D i}\right),
$$

where $V_{D}$ is interface tasks' value for downstream contractors; $P_{D i}$ is the price of downstream interface tasks in interface event $i ; D_{i}$ is the cost for downstream interface tasks normally performed as planned in interface event $i ; W_{D i}$ is the cost for interface conflicts for downstream contractor in interface event $i$. Similarly, $D_{i}$ and $W_{D i}$ are given by:

$$
\begin{gathered}
D_{i}=M_{D i} C_{D i} ; \\
W_{D i}=R_{D i}\left(q_{i}-\overline{H_{i}}\right)=\left(1-\sigma_{i}\right)\left(S_{i}-M_{U i}-M_{D i}\right)\left(q_{i}-\overline{H_{i}}\right),
\end{gathered}
$$

where: $M_{D i}$ - downstream interface tasks performed in interface event $i$ as planned; $C_{D i}-$ unit cost for downstream interface tasks in interface event $i ; R_{D i}$ - downstream reworks caused by interface conflicts in interface event $i ; q_{i}$ - unit cost for reworks for correcting interface conflicts in interface event $i ; \overline{H_{i}}-$ offset unit cost through compensation of interface event $i$. Similarly, the cost for interface conflicts would also arise to downstream contractor if downstream contractor find them and initiate interface meetings to discuss the allocation of responsibilities for the interface conflicts in the latter processes of interface event $i$. The amount of downstream reworks $R_{D i}$ would be negotiated in interface meetings depending on the responsibility allocation for interface conflicts between the two parties and the amount of interface conflicts. The actual unit cost for downstream reworks would also be partly offset through compensation from the owner at the end of the project. 


\subsection{Owner/project manager}

For the owner, the value of interface events is represented by the realization of functional linkages in interface events with the minimum cost. Interface Value, $V_{0}$, for the owner can be detailed as follows:

$$
\begin{gathered}
V_{0}=\sum_{i} \frac{F_{i}}{C_{T i}} \\
C_{T i}=P_{U i}+P_{D i}+\left[\left(S_{i}-M_{U i}-M_{D i}\right)\left(\sigma_{i} H_{i}+\left(1-\sigma_{i}\right) \overline{H_{i}}\right]\right.
\end{gathered}
$$

where: $F_{i}$ - system functional linkages achieved from the interface event $i ; C_{T i}$ - total cost of the entire supply chain in interface event $i$. The interface value analyzes of different participants are based on the assumption that all of the functional linkages could be completed at the end of the project. Without consideration of the owner's requirement changes, $F_{L}$ is a constant value known to the model. Under this assumption, the value of interface events for the owner lies on the minimization of the total cost for all the interface events.

\section{Value Optimization Strategy under status quo delivery methods}

The supply chain actors usually make decisions on their project activities under different scenarios. In this section, firstly, status quo case will be discussed in which each actor aims to receive maximal interface value independently in an adversarial and fragmented supply chain. The result of this scenario is inefficiency of the entire supply chain and loss of Interface Value for all the actors. Then a Value Optimization Strategy and its implementation rules are proposed to shed light on a cooperative and productive supply chain.

\subsection{Status quo case- adversarial and fragmented relations in complex construction projects}

Suppose the actually required interface task $S_{i}$ is uniformly distributed. The probability density function is given by Eqn (9):

$$
\mathrm{f}\left(\mathrm{S}_{i}\right)=\left\{\begin{array}{cr}
\frac{1}{r_{i} p_{i}}, & \text { for } p_{i} \leq \mathrm{S}_{i} \leq\left(1+r_{i}\right) p_{i} \\
0, & \text { elsewhere }
\end{array}\right.
$$

where $p_{i}$ (all the interface tasks indicated in the contracts between owner and the two contractors) and $r_{i}$ (the potential increment in total amount of interface tasks actually required) are considered as constant values. Then the Expected Interface Values for different participants in interface event $i$ are given by Eqns (10), (11) and (12):

$$
\mathrm{E}\left(V_{U i}\right)=P_{U i}-M_{U i} C_{U i}-\sigma_{i}\left(k_{i}-H_{i}\right)\left(\Delta_{i}+\frac{r_{i} p_{i}}{2}\right)
$$

$$
\mathrm{E}\left(V_{D i}\right)=P_{D i}-M_{D i} C_{D i}-\left(1-\sigma_{i}\right)\left(q_{i}-\overline{H_{i}}\right)\left(\Delta_{i}+\frac{r_{i} p_{i}}{2}\right)
$$

$$
\mathrm{E}\left(V_{O i}\right)=\frac{F_{i}}{P_{U i}+P_{D i}+\left[\left(\Delta_{i}+\frac{r_{i} p_{i}}{2}\right)\left(\sigma_{i} H_{i}+\left(1-\sigma_{i}\right) \overline{H_{i}}\right]\right.},
$$

where $\Delta_{i}\left(\Delta_{i}=p_{i}-M_{U i}-M_{D i}\right)$ is the amount of inconsistencies of interface tasks stated in the contracts.

The delivery processes of different subsystems are fragmented and most of the functional linkages of complex construction projects are explicitly manifested in the delivery of disciplines that the downstream contractors are responsible for. As a result, the upstream contractors always make less of an effort to avoid interface conflicts than would be optimal for the entire supply chain. In interface event $i$ under status quo case, the upstream contractor will only complete $m_{i}$ interface tasks which have been clearly stated in contract with the owner before delivering the interface tasks to downstream contractor. While both upstream and downstream contractors focus on eliminating inefficiencies, interface conflicts are usually costlier for the downstream contractors than for the upstream contractors $\left(q_{i}>k_{i}\right)$ because interface conflicts are always recognized during the implementation of downstream interface tasks which means greater costs for downstream contractors to adjust to the variation of the plan. Apart from the downstream interface tasks $n_{i}$ which have been clearly stated in the contract with the owner, downstream contractor will find failures, $S_{i}-m_{i}-n_{i}$ (see Fig. 3), on upstream deliveries which compose of "Interface Conflicts Type I" $\left(\Delta_{i}\right.$, the interface tasks which supposed to be completed by upstream contractor according to the contract between downstream contractor and the owner, ) and "Interface Conflicts Type II" $\left(S_{i}-\Delta_{i}\right.$, the newly appearing interface tasks in the latter stages of interface event $i$ ).

The Expected Interface Values for different participants in status quo case are given by Eqns (13), (14) and (15):

$$
\mathrm{E}\left(V_{U i}\right)=P_{U i}-m_{i} C_{U i}-\sigma_{i}\left(k_{i}-H_{i}\right)\left(p_{i}-m_{i}-n_{i}+\frac{r_{i} p_{i}}{2}\right) ;
$$

$$
\begin{aligned}
\mathrm{E}\left(V_{D i}\right)= & P_{D i}-n_{i} C_{D i}-\left(1-\sigma_{i}\right)\left(q_{i}-\overline{H_{i}}\right) \\
& \left(p_{i}-m_{i}-n_{i}+\frac{r_{i} p_{i}}{2}\right)
\end{aligned}
$$

$\mathrm{E}\left(V_{O i}\right)=$

$\frac{F_{i}}{P_{U i}+P_{D i}+\left[\left(p_{i}-m_{i}-n_{i}+\frac{r_{i} p_{i}}{2}\right)\left(\sigma_{i} H_{i}+\left(1-\sigma_{i}\right) \overline{H_{i}}\right]\right.}$, 


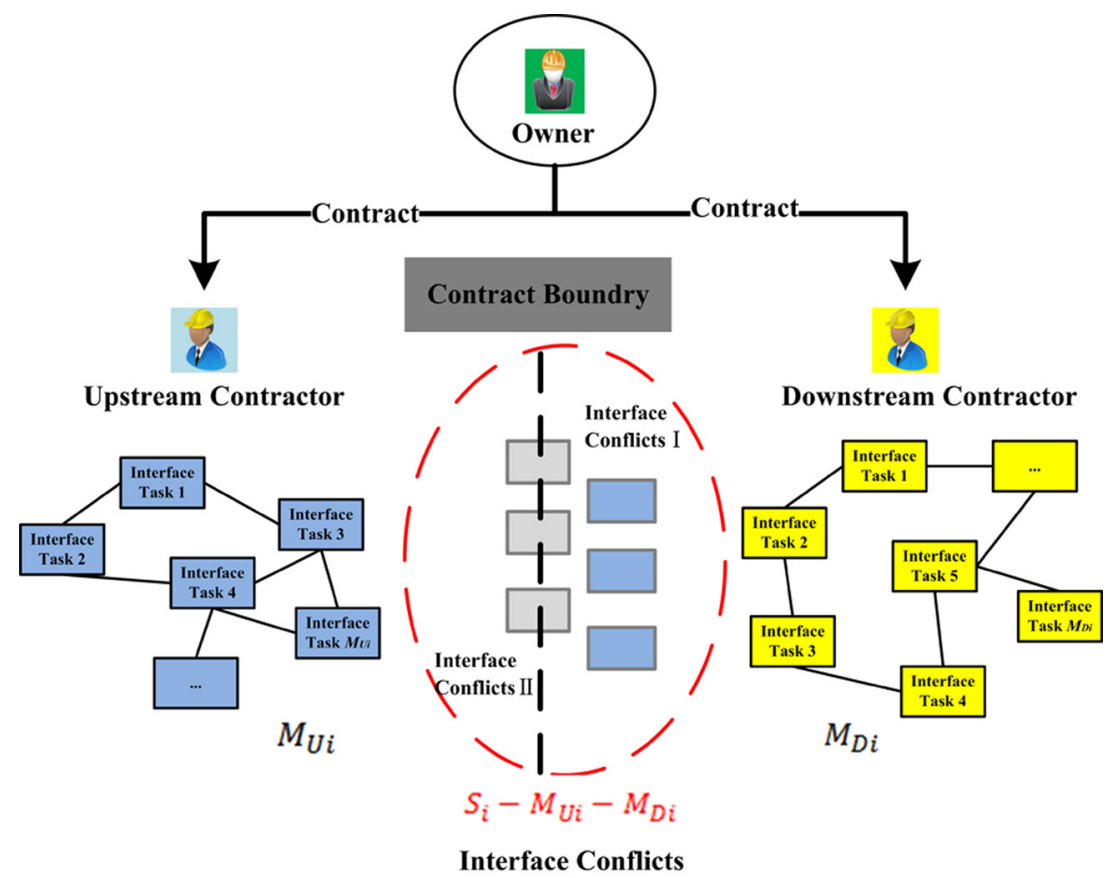

Fig. 3. Two types of interface conflicts

where $\Delta_{i}\left(\Delta_{i}=p_{i}-M_{U i}-M_{D i}=p_{i}-m_{i}-n_{i}\right)$ reaches a maximum in this case.

\subsection{Value Optimization Strategy-cooperative and integrated relations in supply chain}

From the perspective of supply chain management, it would be an optimal scenario under minimal total cost for theentire supply chain which is given by Eqn (16):

$$
\begin{aligned}
C_{T i}= & M_{U i} C_{U i}+M_{D i} C_{D i}+ \\
& \left(S_{i}-M_{U i}-M_{D i}\right)\left[\sigma_{i} k_{i}+\left(1-\sigma_{i}\right) q_{i}\right] .
\end{aligned}
$$

In status quo case the expected value of $C_{T i}$ is given by Eqn (17):

$$
\begin{aligned}
\mathrm{E}\left(C_{T i}\right)= & m_{i} C_{U i}+n_{i} C_{D i}+\left(p_{i}-m_{i}-n_{i}+\frac{r_{i} p_{i}}{2}\right) \\
& {\left[\sigma_{i} k_{i}+\left(1-\sigma_{i}\right) q_{i}\right] . }
\end{aligned}
$$

Interface conflicts identified at latter stages are usually very costly to rectify and inconsistencies in the interface related contract terms are always incurred by the missing of necessary indication of upstream interface responsibilities in the contract between upstream contractor and the owner. Manufacturing Just-in-time (JIT) is a method of pulling work forward from one process to the next "just-in-time", while JIT in construction is to minimize buffers among project activities which are considered as a kind of waste (Ballard, Howell 1995). So without sufficient slack between impacted interface tasks in project schedule, rectifying interface conflicts (variations of the schedule) is costly. Under JIT environ- ment, there are following relations among different types of unit cost: the unit cost for reworks of upstream failures and the newly appearing interface tasks are usually higher than the unit cost for interface tasks as scheduled $\left(k_{i}>C_{U i} ; q_{i}>C_{U i} ; q_{i}>C_{D i}\right)$. So under a common goal of minimizing the total cost, the key is to redistribute the interface responsibilities between the two parties: (1) Unnecessary waste in reworking for $\Delta_{i}$ can be eliminated if upstream contractor could complete them before delivering interface tasks to downstream contractors. (2) The cost for newly appearing interface tasks can be reduced by setting an appropriate value of $\sigma_{i i}$ in interface meetings with having the participant who has lesser cost undertaking the corresponding interface tasks. Then the Expected Interface Values of different participants and entire total cost for supply chain in interface event $i$ are given by Eqns (18), (19), (20) and (21):

$$
\begin{gathered}
\mathrm{E}\left(V_{U i}\right)=P_{U i}-\left(p_{i}-n_{i}\right) C_{U i}-\frac{\sigma_{i} r_{i} p_{i}}{2}\left(k_{i}-H_{i}\right) ;(18) \\
\mathrm{E}\left(V_{D i}\right)=P_{D i}-n_{i} C_{D i}-\frac{r_{i} p_{i}}{2}\left(1-\sigma_{i}\right)\left(q_{i}-\overline{H_{i}}\right) ;(19) \\
\mathrm{E}\left(V_{O i}\right)=\frac{F_{i}}{P_{U i}+P_{D i}+\frac{r_{i} p_{i}}{2}\left[\left(\sigma_{i} H_{i}+\left(1-\sigma_{i}\right) \overline{H_{i}}\right]\right.} ; \quad(20) \\
\mathrm{E}\left(C_{T i}\right)=\left(p_{i}-n_{i}\right) C_{U i}+n_{i} C_{D i}+\frac{r_{i} p_{i}}{2}\left[\sigma_{i} k_{i}+\left(1-\sigma_{i}\right) q_{i}\right] .
\end{gathered}
$$


Comparing the expected interface values for upstream contractor, downstream contractor, owner and the total cost for supply chain in Eqns (18), (19), (20) and (21) of status quo case with that in Eqns (13), (14), (15) and (17) respectively, it shows that the expected interface values of downstream contractor and the owner are higher and the total cost for entire supply chain is less following these redistribution rules on the basis of a cooperative and integrated relations among participants in the supply chain. However, the impact of the redistribution rules on the expected interface value of upstream contractor is not clear without the limitations of correlation between the other variables. This solution would be practical if only each participant could achieve a higher Interface Value in the cooperative supply chain.

\subsection{Implementation rules of Value Optimization Strategy}

Based on the previous analysis, if upstream contractor can be directed to tend to complete inconsistencies in interface tasks $\left(\Delta_{i}\right)$, the value optimization assumption will be practical. The upstream contractor will determine the amount of interface tasks to be finished before delivering them to the downstream contractor based on Eqns (22) and (23):

$$
\begin{aligned}
& V_{U i}=P_{U i}-M_{U i} C_{U i}-\sigma_{i}\left(k_{i}-H_{i}\right)\left(S_{i}-M_{U i}-n_{i}\right) ; \\
& \mathrm{E}\left(V_{U i}\right)=[\left.\sigma_{i}\left(k_{i}-H_{i}\right)-C_{U i}\right] M_{U i}- \\
& \sigma_{i}\left(k_{i}-H_{i}\right)\left(p_{i}-n_{i}+\frac{r_{i} p_{i}}{2}\right)+P_{U i},
\end{aligned}
$$

where: $m_{i} \leq M_{u i} \leq p_{i}-n_{i}$. If $\sigma_{i}\left(k_{i}-H_{i}\right)-C_{U i}>0$, the upstream contractor would set $M_{U i}$ to be $p_{i}-n_{i}$ which is the best case with $\Delta_{i}=0$, however if $\sigma_{i}\left(k_{i}-H_{i}\right)-C_{U i}<0$, the upstream contractor would set $M_{U i}$ to be $m_{i}$ which is the worst case with $\Delta_{i}=p_{i}-m_{i}-n_{i}$.

A practical strategy with a less cost for the entire supply chain and optimal value for each of the participants can be realized by dealing with the two types of interface conflicts separately and results in the elimination of Interface Conflicts Type I. The extreme value of $\sigma_{i}$ (proportion of responsibilities for interface conflicts claimed from owner and upstream contractor in interface meetings for interface event $i$ ) and $H_{i}$ (offset unit cost through compensation in interface event $i$ ) are the core of this strategy. A penalty term is added to the contract of upstream contractor to restrict the behaviors of upstream contractor stating that "if $M_{u i}<p_{i}-n_{i}$, then setting $\sigma_{i}=1$ and $H_{i}=0$ ". In other words, if the upstream contractor cannot complete all the necessary interface tasks required by downstream contractors before the delivering point, then they have to rework all the missing interface tasks at a higher cost $k_{i}$ without any compensation as a penalty. In this situation, $\sigma_{i}\left(k_{i}-H_{i}\right)-C_{U i}=k_{i}-C_{U i}>0$, and upstream contractor will determine to complete $p_{i}-n_{i}$ interface tasks before the delivering point to maximize the expected interface value. However, the Interface Conflicts Type II are still inevitable under this strategy and all the participants should allocate responsibility allocation solution for the newly appearing interface tasks in the principle of minimizing the cost for entire supply chain. In addition, the previous analysis are all based on the assumption that all the participants share necessary information about the value of $p_{i}, m_{i}$ and $n_{i}$ so an improved approach in eliminating Interface Conflicts Type II under productive cooperation environment is essential.

\section{Prospects of IPD in eliminating interface conflicts}

Fragmentation in delivery processes of subsystem contractors in complex construction projects is the root cause of interface conflicts and inefficiencies of supply chain. Hence, improvements in procurement procedures are desirable. In this section, prospects for application of IPD on supply chain integration practices are discussed. IPD is defined by American Institute of Architects (AIA) as "a project delivery approach that integrates people, systems, business structures, and practices into a process that collaboratively harnesses the talents and insights of all project participants to optimize project results, increase value to the owner, reduce waste, and maximize efficiency through all phases of design, fabrication, and construction". Kent and Becerik-Gerber (2010) define IPD with the following principles: (1) multiparty agreements (MPA); (2) early involvement of all parties; and (3) shared risk and reward. Based on these principles, the status quo case described in Section 5 can be improved by using IPD.

\subsection{IPD-based scenario}

In IPD procurement/delivery system, MPA take place where the primary project participants execute a single contract specifying their roles, rights, obligations, and liabilities (Marzouk, Elmesteckawi 2015) (see Fig. 4). Under this contract, the multi-disciplinary participants have common goals and interest and have to coordinate with each other to optimize the expected value of the entire supply chain. Opportunities for supply chain integration are increased with IPD where the functional disciplines can be brought early into the project together and evaluate the effect of interface events on the performance of the entire construction supply chain. Only by optimizing the expected value of the entire supply chain could they achieve their own maximal profit. The IPD contract links the risks and rewards of all the participants with the common goals of the holistic project. For example, if the interface tasks of a subsystem contractor are delayed or negatively impacted by any factors, the cost for waste in solving these problems has to be undertaken by all the related subsystem contractors. Even without mandatory interface management policies pushed ahead by the owner, 


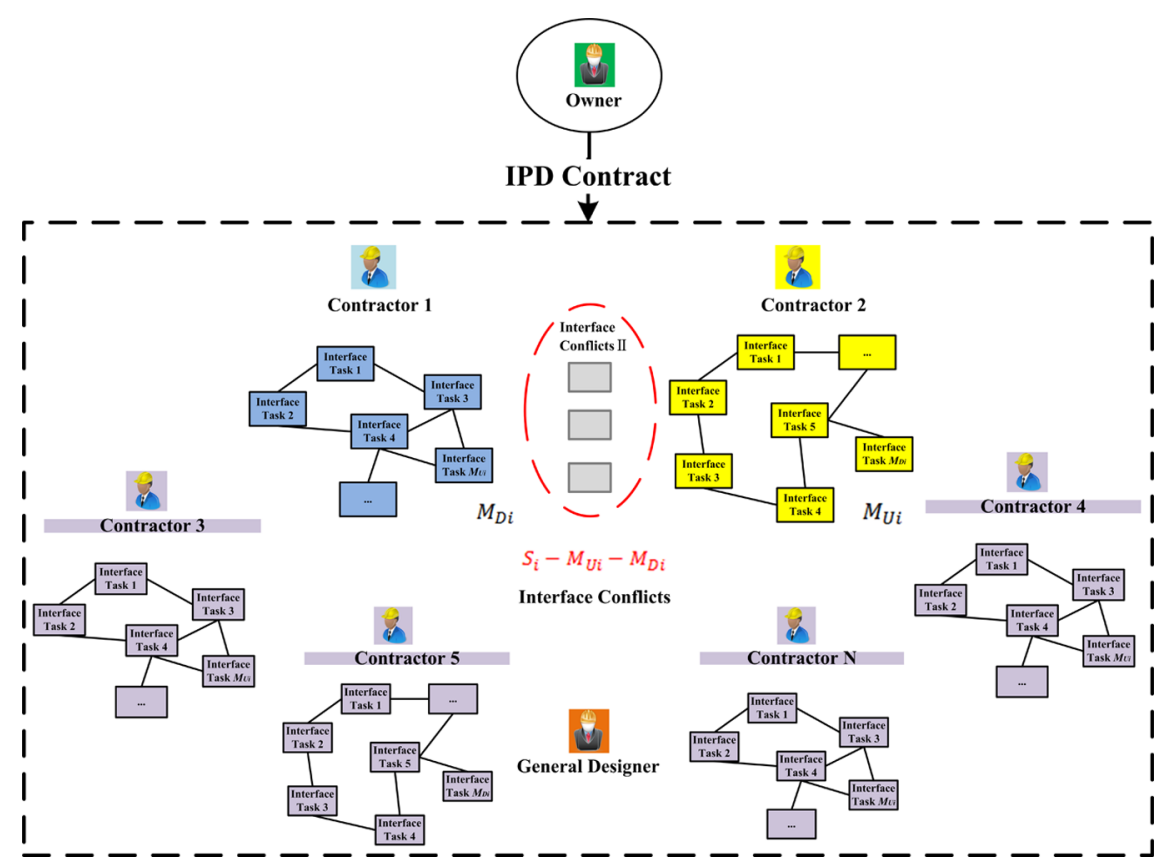

Fig. 4. IPD contract expected to eliminate interface conflicts

the associated participants will hold interface meetings frequently in early phases of the project and downstream contractors will be involved in the early implementation of upstream interface tasks to avoid interface conflicts to the outmost extent in late stages of the project which are costly to fix.

\subsection{IPD-based interface value model}

Under IPD environment, Interface Conflicts Type Icaused by inconsistencies of interface tasks in different contracts can be directly removed owing to the unified IPD contract and there only exits Interface Conflicts Type IIin this scenario. Interface Conflicts Type II (the newly appearing interface tasks in the latter stages of interface event i) are usually closely related to the gaps between system's general design, subsystems' detailed design and manufacturing, as well as insufficient communication among participants in the early project phases. As a result, technically, Interface Conflicts Type II cannot be completely predicted in early stages of the project. However, shared risks and early involvement create a positive atmosphere of the supply chain. Interface Conflicts Type II will be expected to significantly decrease in interface events. "Incentive pool" is one of the risks/rewards sharing methods in IPD (Kent, Becerik-Gerber 2010). It is implemented by reserving a unique portion of the related participant's expenses into a pool that can be distributed to the participants based on their project performances. The Expected Interface Values for upstream and downstream contractor in interface event $i$ are given by Eqns (24) and (25):

$$
\begin{aligned}
\mathrm{E}\left(V_{U i}\right)= & P_{U i}\left(1-a_{i}\right)-\left(p_{i}-n_{i}\right) C_{U i}-\frac{\sigma_{i} r_{i} p_{i}}{2}\left(k_{i}-H_{i}\right)+ \\
& \omega_{i}\left(a_{i} P_{U i}+b_{i} P_{D i}\right) ;
\end{aligned}
$$

$$
\begin{aligned}
\mathrm{E}\left(V_{D i}\right)= & P_{D i}\left(1-b_{i}\right)-n_{i} C_{D i}-\frac{r_{i} p_{i}}{2}\left(1-\sigma_{i}\right)\left(q_{i}-\overline{H_{i}}\right)+ \\
& \left(1-\omega_{i}\right)\left(a_{i} P_{U i}+b_{i} P_{D i}\right),
\end{aligned}
$$

where: $\omega_{i}$ - proportion of upstream interface tasks value achieved by upstream contractor from the incentive pool in interface event $i ; a_{i}$-proportion of upstream expenses reserved into the "incentive pool" in interface event $i$; $b_{i}$ - proportion of downstream expenses reserved into the "incentive pool" in interface event $i$. The expressions of $\mathrm{E}\left(V_{O i}\right)$ and $\mathrm{E}\left(C_{T i}\right)$ are the same as Eqns $(20)$ and (21), respectively, in the Value Optimization Strategy under status quo delivery methods.

\subsection{IPD-based approach vs. Value Optimization Strategy vs. status quo case}

Comparing the Expected Interface Value of upstream and downstream contractor in Eqns (24) and (25) of the IPD approach with that in Eqns (18) and (19) of the Value Optimization Strategy under the status quo project delivery method, it is evident that both contractors have relatively lower cost in dealing with interface conflicts because of the elimination of "Interface Conflicts Type I". The differences lie in the cost for "Interface Conflicts Type II". The introduction of an "Incentive Pool" leads to a shared-risks environment which means the contractors have to productively cooperate with each other to control the risks of "Interface Conflicts Type II" by eliminating these conflicts as early as possible and preventing them from escalating into costly problems. The owner will allocate the profit in the incentive pool for improved project performance of related contractors. The most direct manifestation of success would be the decline in unit cost for dealing with "Interface 
Conflicts Type II" of both upstream $\left(k_{i}\right)$ and downstream contractor $\left(q_{i}\right)$. Similarly, offset unit cost in compensation $H_{i}$ and $\overline{H_{i}}$, which have a positive correlation with $k_{i}$ and $q_{i}$ will decrease also. As seen from Eqns (20) and (21), the expected interface value for the owner and the total cost for supply chain are improved as well with a lower values of $k_{i}, q_{i}, H_{i}$ and $\overline{H_{i}}$. It should be noted that "Interface Conflicts Type I" cannot be completely eliminated in the Value Optimization Strategy in practice because under the status quo delivery method the upstream contractor would not have complete knowledge of $p_{i}$ and $n_{i}$ as assumption. In conclusion, compared with status quo case, Value Optimization Strategy reduces most of the Interface Conflicts Type I and the average unit cost for Interface Conflicts Type II while IPD-based approach eliminates Interface Conflicts Type Iand tends to significantly reduce Interface Conflicts Type II. Figure 5 shows the trends of expected costs for the two types of interface conflicts in the three scenarios.

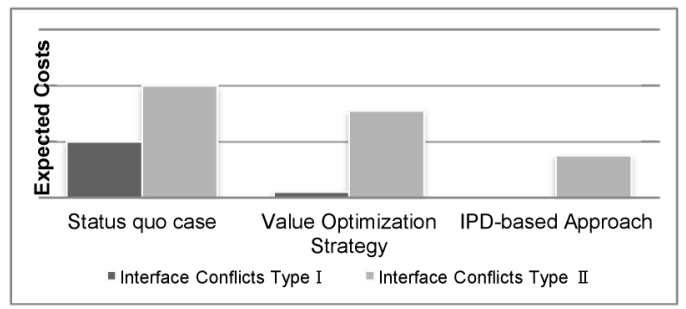

Fig. 5. Comparison of expected cost for interface conflicts in different scenarios

\section{Pilot study}

This section will demonstrate Value Optimization Strategy under status quo delivery methods and IPD-based approach by pilot study in real projects.

\subsection{Background}

The pilot study is based on three metro construction projects in China which involve multi-disciplinary subsystems such as tunnels, metro stations, vehicles, rails, telecommunications, signal, powers, escalators, lighting, etc. Besides, a variety of equipment automatic control systems have been applied such as Integrated Supervisory Control Systems (ISCS), Building Automation Systems (BAS), Fire Alarm Systems (FAS), etc. As an integrated monitoring platform, Integrated Supervisory Control Systems (ISCS) has tens of thousands of physical interfaces with other subsystems (including Building Automation Systems), leaving it as one of the most important and complex objects of Interface Management. ISCS contractors, BAS contractors and owners of the metro projects are chosen as the participants for pilot study. Helishi is a Chinese company specialized in automatic control system design, manufacture and construction, including ISCS and BAS of metro projects. For the sake of comparison and evaluation, the pilot study is conducted in three metro projects in China with Helishi serving as the ISCS contractor.

Case A: Case A is based on the history data of a Chinese metro project under status quo delivery methods which has been built three years ago. A typical interface event between ISCS and BAS with frequent interface conflicts happening in the latter stages of the project is chosen. Within range of the interface event, the upstream contractor is BAS contractor and the downstream contractor is ISCS contractor (Helishi).

Case B: Case B is based on a metro project under construction in southern China under status quo delivery methods. Based upon project experience, the owner and Helishi find it difficult to eliminate interface conflicts among multi-disciplinary subsystem contractors which always lead to unnecessary wastes and reworks. Value Optimization Strategy is implemented in the same interface event as Case A among the owner, BAS contractor and ISCS contractor for pilot study. Similarly, the upstream contractor is a BAS contractor and the downstream contractor is ISCS contractor (Helishi).

Case C: Although Integrated Project Delivery (IPD) has been demonstrated as an effective delivery method in academic field, it still has not been promoted in practice in China. The reasons for the barriers of IPD application are comprehensive, one of which is the lack or inadequacy of necessary laws and regulations. Case $\mathrm{C}$ is also based on a pilot project under status quo delivery methods. But its uniqueness lies in that Helishi undertake the design, manufacture and construction of both BAS and ISCS of the pilot project which means we have a similar delivery condition to IPD in this case. The same interface event as in Case A and Case B is chosen. Both of the upstream contractor and downstream contractor are Helishi.

\subsection{Case modelling}

Case A: Let us firstly consider the status quo case serving as the control group for validation of Value Optimization Strategy in Case B and IPD-based approach in Case C. According to the history data of the typical interface event between Integrated Supervisory Control Systems (ISCS) and Building Automation Systems (BAS), the parameters are specified as follows: The price of upstream interface tasks in the interface event is $P_{U i}=\$ 1,200$. The upstream interface tasks performed before delivering to downstream contractor which have been clearly stated in contract is $m_{i}=20$. The unit cost for upstream interface tasks is $C_{U i}=\$ 45$ per unit. The proportion of responsibilities for interface conflicts claimed from owner and upstream contractor in interface meetings is $\sigma_{i}=0.6$. The unit cost for upstream reworks for interface conflicts is $k_{i}=\$ 50$ per unit. The offset unit cost for upstream contractor in compensation is $H_{i}=\$ 35$ per unit. All the interface tasks indicated in the contracts between owner and the two contractors is $p_{i}=54$. The potential increment in total amount of interface tasks actually required is $r_{i}=0.14$. The price of downstream interface tasks in 
the interface event is $P_{D i}=\$ 1,600$. The downstream interface tasks performed which have been clearly stated in contract is $n_{i}=30$. The unit cost for downstream interface tasks is $C_{D i}=\$ 38$ per unit. The unit cost for downstream reworks for interface conflicts is $q_{i}=\$ 55$ per unit. The offset unit cost for downstream contractor in compensation is $\overline{H_{i}}:=\$ 40$ per unit. Then the Expected Interface Value for upstream contractor is $\mathrm{E}\left(V_{U i}\right)=229.98$ (see Eqn (13)). The Expected Interface Value for downstream contractor is $\mathrm{E}\left(V_{D i}\right)=413.32$ (see Eqn (14)). We also get the expected total cost for the entire supply chain in the interface event, that is $\mathrm{E}\left(C_{T i}\right)=2444.56$ (see Eqn (17)).

Case B: Case B is established to demonstrate Value Optimization Strategy in industry. We select the same interface event of a similar metro project as in Case A, so the values of constant parameters of Case B are close to the ones in Case A. For the sake of comparison, the values of the following parameters of Case $\mathrm{A}$ and Case $\mathrm{B}$ are considered as the same. The price of upstream interface tasks in the interface event is $P_{U i}=\$ 1,200$. The upstream interface tasks which have been clearly stated in contract is $m_{i}=20$. The unit cost for upstream interface tasks is $C_{U i}=\$ 45$ per unit. The unit cost for upstream reworks for interface conflicts is $k_{i}=\$ 50$ per unit. All the interface tasks indicated in the contracts between owner and the two contractors is $p_{i}=54$. The potential increment in total amount of interface tasks actually required is $r_{i}=0.14$. The price of downstream interface tasks in the interface event is $P_{D i}=\$ 1600$. The downstream interface tasks performed which have been clearly stated in contract is $n_{i}=30$. The unit cost for downstream interface tasks is $C_{D i}=\$ 38$ per unit. The unit cost for downstream reworks for interface conflicts is $q_{i}=\$ 55$ per unit. Two rounds of three-party interface meetings are initiated at very beginning of the interface event to confirm upstream interface tasks. Four interface tasks $\left(p_{i}-m_{i}-n_{i}=4\right)$ which should be completed by upstream contractor are identified in interface meetings. In the contract with upstream contractor, a penalty item is stated: if the upstream contractor cannot complete all the necessary interface tasks identified in the three-party interface meetings before delivering points, there will be no compensation for upstream reworks. At the end of the interface event, it is manifested that the inconsistencies of interface tasks stated in the contracts are eliminated $\left(\Delta_{i}=p_{i}-M_{U i}-M_{D i}=0\right)$. However, the newly appearing interface conflicts happening during the implementation of downstream interface tasks still lead to reworks and wastes. According to Value Optimization Strategy, the cost for newly appearing interface tasks can be reduced by setting an appropriate value of $\sigma_{i}$ with having the participant who has lesser cost undertaking the corresponding interface tasks. In other words, it would be an optimal case setting $\sigma_{i}=1$ and allocating all the newly appearing interface tasks to BAS contractor. However, in Case B, because the cost for fixing interface conflicts are usually higher than compensation achieved from the owner, BAS contractor is reluctant in reworks and the three parties argue back and forth in interface meetings for the determination of $\sigma_{i}, H_{i}$ and $\overline{H_{i}}$ which may differ from case to case. For the simplicity of comparison, we use the same values of $\sigma_{i}, H_{i}$ and $\overline{H_{i}}$ as Case A in modeling Case $\mathrm{B}$. The proportion of responsibilities for interface conflicts claimed from owner and upstream contractor in interface meetings is $\sigma_{i}=0.6$. The offset unit cost for upstream contractor in compensation is $H_{i}=\$ 35$ per unit. The offset unit cost for downstream contractor in compensation is $\overline{H_{i}}:=\$ 40$ per unit. Then the Expected Interface Value for upstream contractor is $\mathrm{E}\left(V_{U i}\right)=85.98$ (see Eqn (18)). The Expected Interface Value for downstream contractor is $\mathrm{E}\left(V_{D i}\right)=437.32$ (see Eqn (19)). The expected total cost for the entire supply chain in the interface event is $\mathrm{E}\left(C_{T i}\right)=2416.56$ (see Eqn (21)).

Case C: Case C is modelled to demonstrate IPDbased approach. Helishi undertake the design, manufacture and construction of both ISCS and BAS in Case C. In the range of the selected interface event, the owner, upstream contractor and downstream contractor execute a single contract specifying their roles, rights, obligations and liabilities (see Fig. 4). Interface Conflicts Type I caused by inconsistencies of interface tasks in different contracts do not exist in this scenario. At the very beginning of pilot project, optimal design, manufacture and construction of the interfaces between BAS and ISCS are taken into consideration as viewed from the holistic metro system and all the participants share the full knowledge of interface information with common goals during the implementation of the interface event. Interface meetings are hold frequently to reduce the potential risks of newly appearing interface conflicts which are usually costly to fix in late stages of the interface event. Based on the same interface event as Case A and Case B with similar infective factors of $r_{i}$, the distinct advantage of Case $\mathrm{C}$ lies in the obvious decline of $r_{i}\left(r_{i}=0.08\right)$. In other words, although Interface Conflicts Type II cannot be completely predicted in early stages of the pilot project, the uncertainty of interface tasks has been significantly reduced in this case. The upstream contractor and downstream contractor are the same company, so "Incentive pool" is not applicable in Case C. Then the Expected Interface Value for upstream contractor is $\mathrm{E}\left(V_{U i}\right)=100.56$ (see Eqn (18)). The Expected Interface Value for downstream contractor is $\mathrm{E}\left(V_{D i}\right)=447.04$ (see Eqn (19)). The expected total cost for the entire supply chain in the interface event is $\mathrm{E}\left(C_{T i}\right)=2332.32$ (see Eqn (21)).

\subsection{Comparisons and limitations}

The results of modeling the three cases are summarized in Figure 6. The expected total cost for the entire supply chain in Case B is $2 \%$ less than Case A, while the expected total cost for the entire supply chain in Case $\mathrm{C}$ is $5 \%$ less than Case A. Meanwhile, the Expected Interface Value for downstream contractor is in Case B is $6 \%$ higher 
than Case A and the Expected Interface Value for downstream contractor in Case $\mathrm{C}$ is $9 \%$ higher than Case A. However, it is also manifested that the Expected Interface Value for upstream contractor is in Case B is 63\% lower than Case A and the Expected Interface Value for downstream contractor in Case C is 57\% lower than Case A. In summary, the performance of Value Optimization Strategy and IPD-based approach are validated by the cost savings of entire supply chain with upstream contractor bearing the losses. Meanwhile however, in practice the upstream contractors in interface events would probably be reluctant to cooperate and the implementation of these two strategies would have the chance to fail.

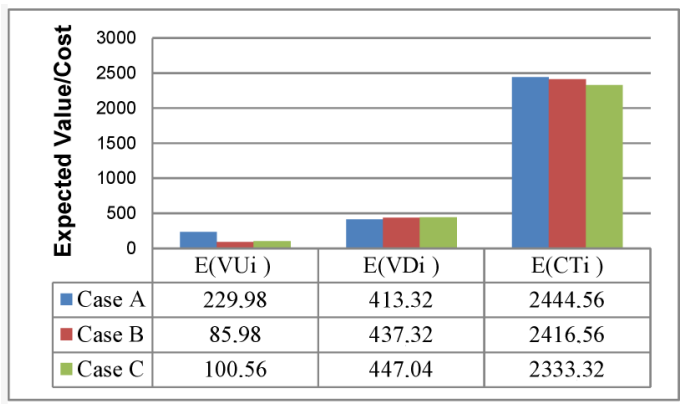

Fig. 6. Results of modelling the three cases

Parameter $r_{i}$ describes the uncertainty level of interface tasks and potential risks of Interface Conflicts Type II. We use the predicted value of $r_{i}$ in the modeling of three cases based on project experience. Based on the actual values of $\mathrm{S}_{i}$ (actually required interface tasks in interface event $i$ ), $V_{U i}$ (Interface Value for upstream contractor), $V_{D i}$ (Interface Value for downstream contractor) and $C_{T i}$ (total cost of the entire supply chain in interface event $i$ ) are calculated by upstream contractors, downstream contractors and owners of the three cases based on Eqns (1), (4) and (8), respectively (see Table 1). The gaps between predicted data and actual data mainly depend on the accuracy of predictions of Interface Conflicts Type II. Comparing the data in Table 1 and Figure 6, it can be observed that under status quo delivery method Interface Conflicts Type II may lead to more reworks and wastes than expected. However, in IPD scenario nearly 75\% Interface Conflicts Type II has been eliminated because shared risks and early involvement create a positive atmosphere of the supply chain.

Table 1. Results of pilot study based on actual data

\begin{tabular}{lccc}
\hline & $V_{U i}$ & $V_{D i}$ & $C_{T i}$ \\
\hline Case A & 210.72 & 400.48 & 2555.84 \\
Case B & 66 & 424 & 2532 \\
Case C & 106.5 & 451 & 2298 \\
\hline
\end{tabular}

Moreover, comparing project behaviors of multidisciplinary participants through interface meetings and interviews in the interface events of three cases, we found that there are different attitude toward the implementation of the new strategies among upstream contractors, downstream contractors and owners. Helishi had interfaces with almost all the other subsystems and IM played a vital role in functional realization of ISCS itself. So IM goals of ISCS tend to be the same as the owners. Their common benefits and values make them put more emphasis on IM compared with other participants in supply chain which are more independent and have fewer interface problems. The downstream contractors in Case B and Case C are reluctant in the implementation of the new strategies and the success of pilot study is based on their cooperation which is limited in the range of the typical interface event we selected. We conclude that: (1) The cooperation of upstream contractors directly affects the elimination of Interface Conflicts Type I. (2) Value Optimization Strategy would have chance to fail if the project behaviors of upstream contractor in supply chain are not well organized and controlled during project implementation. (3) IPD-based approach which creates common goals and interest in a single contract for multi-disciplinary participants achieves the optimal performance in eliminating both Interface Conflicts Type I and Interface Conflicts Type II in complex supply chains. Although this research provides exposure to the conflicting behaviors of multidisciplinary participants of complex supply chains from an interface management based perspective and propose approaches to eliminate unnecessary interface conflicts which have been proved to be useful in practice, it has some limitations. (1) The upstream contractors which are relatively independent in the entire metro system do not value IM and tend to be reluctant to cooperate in interface tasks. The results of pilot study are limited to the typical interface event. Value Optimization Strategy under status quo delivery method may have chance to fail in other projects. (2) The upstream contractor and downstream contractor in Case $\mathrm{C}$ are the same company. The differences between project condition of Case $\mathrm{C}$ and a real IPD project may lead to deviations of the evaluation of IPDbased approach. (3) For the sake of comparisons, we ignore the tiny differences of values of the basic parameters in the same interface events of three cases in pilot study. Overall, the result of pilot study demonstrates that Value Optimization Strategy and IPD-based approach are useful and effective ways to eliminate interface conflicts and avoid unnecessary reworks and wastes. IM performance can be improved among multi-disciplinary participants in complex supply chains of construction projects.

\section{Conclusions and future research}

SCM and IM should still be considered as immature practices in complex construction projects. SCM concepts from manufacturing sector may not be directly applicable in construction. IM cannot be precisely defined because the definition of "Interface" is broad and it relies on artificial divisions of project management functional components in different segments of the industry. This 
paper described strategies to eliminate interface conflicts on the boundaries between multiple prime contractors and to improve supply chain integration of complex construction projects characterized by adversarial short-term relationships and fragmentations in project delivery procedures. Two types of interface conflicts are recognized and defined within the scope of this research. The paper describes "interface events" and "interface tasks" as a foundation for solving interface issues between multidisciplinary participants. We evaluated the value-driven behaviors of different participants and how behaviors of one discipline impact on the related disciplines by determining the expected interface values achieved in the same interface events. The value optimization strategy presented above under status quo delivery methods reduced "Interface Conflicts Type I" and achieved relatively lower cost of the entire supply chain. Improvements in procurement procedures are the key solutions to the two types of interface conflicts. IPD-based approaches further eliminated "Interface Conflicts Type II" as well as "Interface Conflicts Type I", integrated the complex supply chain and reduced unavoidable waste and potential risks for delivering required customer value on time. There are practical implications. The paper is expected to benefit the owner of complex construction projects in understanding the root causes of conflicting behaviors among prime contractors. Value Optimization Strategy along with IPD-based approach provides IM proposals to eliminate interface conflicts and integrate the complex supply chain in practice. The owners of complex construction projects need to pay greater attention to the integration practices of the supply network and coordinate the interfaces between multiple prime contractors which might significantly save project cost and duration.

This research proposes approaches to eliminate interface conflicts in complex construction projects. Further research studying implementation principles of applying IPD on IM from theoretical and practical perspective is essential. Moreover, Value Optimization Strategy and IPD-based approach should be implemented based on participants' complete knowledge of interface information and clear understanding of interface responsibilities and priorities. Proactive interface identification (identifying interface events and scheduling interface tasks) is the key to success of interface management and complex supply chain integration. An interface mapping and tracking approach should be promoted in future research in order to provide essential interface knowledge for key participants in the complex construction projects and to visualize potential interface risks during the whole lifecycle of project. Graph theory is one of the structured modeling approaches to provide a formal mathematical framework and computer-based environment. It enables model-based work to be done productively. An "Interface Graph" should be explored in future research to visualize and analyze explicit/implicit complex interface relationships, to help eliminate potential interface conflicts and to further improve supply chain integration of complex construction projects.

\section{Acknowledgement}

Qianqian Ju gratefully acknowledges China Scholarship Council (Grant No. 201406160066) for funding a joint doctoral fellowship between Huazhong University of Science and Technology and University of Maryland, College Park.

\section{References}

Al-Hammad, A.-M. 1995. Interface problems between owners and maintenance contractors in Saudi Arabia, Journal of Performance of Constructed Facilities 9(3): 194-205. https://doi.org/10.1061/(ASCE)0887-3828(1995)9:3(194)

Al-Hammad, A.-M. 2000. Common interface problems among various construction parties, Journal of Performance of Constructed Facilities 14(2): 71-74. https://doi.org/10.1061/(ASCE)0887-3828(2000)14:2(71)

Arantes, A.; Ferreira, L. M. D. F.; Costa, A. A.; Wagner, B. 2015. Is the construction industry aware of supply chain management? The Portuguese contractors' perspective, Supply Chain Management: An International Journal 20(4): 404-414.

AS/NZS 4183: 1994. Value management. Australia and New Zealand Standard, 1994.

Ballard, G.; Howell, G. 1995. Toward construction JIT, Lean Construction 2: 291-300.

Dainty, A. R. J.; Briscoe, G. H.; Millett, S. J. 2001. Subcontractor perspectives on supply chain alliances, Construction Management and Economics 19(8): 841-848. https://doi.org/10.1080/01446190110089727

Emmitt, S.; Sander, D.; Christoffersen, A. K. 2005. The value universe: defining a value based approach to lean construction, in International Group on Lean Construction, 2005, Sydney, Australia, 57-64.

Fearne, A.; Fowler, N. 2006. Efficiency versus effectiveness in construction supply chains: the dangers of "lean" thinking in isolation, Supply Chain Management: An International Journal 11(11): 283-287.

Gunasekaran, A.; Love, P. E. D. 1998. Concurrent engineering: a multi-disciplinary approach for construction, Logistics Information Management 11(5): 295-300. https://doi.org/10.1108/09576059810234209

Harrison, P. 2004. An overview of interface management, in ASME/IEEE 2004 Joint Rail Conference, 8 April 2004, Baltimore, MD, USA, 89-99.

Humphreys, P.; Matthews, J.; Kumaraswamy, M. 2003. Preconstruction project partnering: from adversarial to collaborative relationships, Supply Chain Management: An International Journal 8(2): 166-178.

Ju, Q.; Ding, L. 2014. A web-based system for interface management of metro equipment engineering, Journal of Intelligent \& Robotic Systems 79(3-4): 577-590.

Kelly, B.; Berger, S. 2006. Interface management: effective communication to improve process safety, Journal of Hazardous Materials 130(3): 321-325. https://doi.org/10.1016/j.jhazmat.2005.07.009

Kent, D. C.; Becerik-Gerber, B. 2010. Understanding construction industry experience and attitudes toward integrated project delivery, Journal of Construction Engineering and Management 136(8): 815-825. https://doi.org/10.1061/(ASCE)CO.1943-7862.0000188

Lin, Y.-C. 2013. Construction network-based interface management system, Automation in Construction 30: 228-241. https://doi.org//10.1016/j.autcon.2012.11.025 
Love, P. E.; Irani, Z.; Edwards, D. J. 2004. A seamless supply chain management model for construction, Supply Chain Management: An International Journal 9(1): 43-56.

Love, P. E. D.; Gunasekaran, A. 1997. Concurrent engineering in the construction industry, Concurrent Engineering: Research and Applications 5(2): 155-62. https://doi.org/10.1177/1063293X9700500207

Marzouk, M.; Elmesteckawi, L. 2015. Analyzing procurement route selection for electric power plants projects using SMART, Journal of Civil Engineering and Management 21(7): 912-922. https://doi.org/0.3846/13923730.2014.971131

Nooteboom, U. 2004. Interface, management improves on-time, on-budget delivery of megaprojects, Journal of Petroleum Technology 56(8): 32-34. https://doi.org/10.2118/0804-0032-JPT

Pavitt, T.; Gibb, A. 2003. Interface management within construction: In particular, building façade, Journal of Construction Engineering and Management 129(1): 8-15. https://doi.org/10.1061/(ASCE)0733-9364(2003)129:1(8)

Segerstedt, A.; Olofsson, T.; Bankvall, L.; Bygballe, L. E.; Dubois, A.; Jahre, M. 2010a. Interdependence in supply chains and projects in construction, Supply Chain Management: An International Journal 15(5): 385-393.

Segerstedt, A.; Olofsson, T.; Eriksson, P. E. 2010b. Improving construction supply chain collaboration and performance: a lean construction pilot project, Supply Chain Management: An International Journal 15(5): 394-403.
Senthilkumar, V.; Varghese, K. 2012. Case study-based testing of design interface management system, Journal of Management in Engineering 29(3): 279-288. https://doi.org/10.1061/(ASCE)ME.1943-5479.0000150

Senthilkumar, V.; Varghese, K.; Chandran, A. 2010. A webbased system for design interface management of construction projects, Automation in Construction 19(2): 197-212. https://doi.org/10.1016/j.autcon.2009.10.007

Siao, F.-C.; Lin, Y.-C. 2012. Enhancing construction interface management using multilevel interface matrix approach, Journal of Civil Engineering and Management 18(1): 133-144. https://doi.org/10.3846/13923730.2012.657368

Turkulainen, V.; Ruuska, I.; Brady, T.; Artto, K. 2015. Managing project-to-project and project-to-organization interfaces in programs: Organizational integration in a global operations expansion program, International Journal of Project Management 33(4): 816-827. https://doi.org/10.1016/j.ijproman.2014.10.008

Whyte, J.; Stasis, A.; Lindkvist, C. 2016. Managing change in the delivery of complex projects: configuration management, asset information and 'big data', International Journal of Project Management 32(2): 339-351. https://doi.org/10.1016/j.ijproman.2015.02.006

Wideman, M. 2002. Wideman comparative glossary of project management terms v3.1.

Qianqian JU. She is a PhD student of Huazhong University of Science and Technology, Wuhan (Hubei), China. She is also a joint $\mathrm{PhD}$ student of Construction Engineering at the Center of Excellence in Project Management in the A. J. Clark School of Engineering the at the University of Maryland, College Park (USA) sponsored by China Scholarship Council. Her primary research interests include project management, supply chain management, interface management, integrated project delivery and process control.

Lieyun DING. PhD, is a professor, director of the BIM Research Laboratory and rector/president of Huazhong University of Science and Technology, Wuhan (Hubei), China. He has previously served as rector/president of Northeastern University in Shenyang (Liaoning), China. His research interests focus on applying information and automation technologies to construction engineering and management, and on safety and risk management for large-scale built infrastructure, including metro systems, bridges and tunnels. Professor Ding is the editor-in-chief of the Chinese Journal of Civil Engineering and Management. He also serves as deputy director of the Management Division of Science and Technology Committee of China's Ministry of Education, chairman of the Architectural Society of China Construction Management Research Division, deputy chairman of the Chinese Society of Metals, and director of China's National Accreditation Board for Construction Management.

Miroslaw J. SKIBNIEWSKI. He is a Professor of Construction Engineering at the Center of Excellence in Project Management in the A. J. Clark School of Engineering the at the University of Maryland, College Park (USA). He holds his Master of Eng. in Civil Engineering from Warsaw University of Technology (Poland) and MSc and $\mathrm{PhD}$ degrees from Carnegie-Mellon University, Pittsburgh, Pennsylvania (USA). He is an author or co-author of over 240 publications on a broad range of topics related to engineering project management, information technology and automation engineering applied in construction and other project-based industries. He is a recipient of numerous U.S. and international research awards, including an honorary doctorate from Vilnius Gediminas Technical University (Lithuania). 